\title{
Current Status of Researches on Surveillance and Early Warning of Mental Health
}

\author{
Lingjiang Liu, Guolin Bian* \\ Ningbo Kangning Hospital, Ningbo, China \\ Email: 179294022@qq.com, *niuniu0815@126.com
}

How to cite this paper: Liu, L.J. and Bian, G.L. (2019) Current Status of Researches on Surveillance and Early Warning of Mental Health. Open Access Library Journal, 6: e5832.

https://doi.org/10.4236/oalib.1105832

Received: October 6, 2019

Accepted: October 20, 2019

Published: October 23, 2019

Copyright $\odot 2019$ by author(s) and Open Access Library Inc.

This work is licensed under the Creative Commons Attribution International License (CC BY 4.0).

http://creativecommons.org/licenses/by/4.0/

\section{(c) () Open Access}

\begin{abstract}
In this article, we reviewed and analyzed the literature on mental health surveillance and early warning of mental disorders, in particular, systematically reviewed the progress and methods of mental health surveillance and early warning of mental disorders. This article provides a basis for early warning and monitoring of mental disorders in the future, and provides psychiatrists and community doctors with reference methods for early warning and treatment of mental disorders so as to ensure social stability and harmony.
\end{abstract}

\section{Subject Areas}

Psychiatry \& Psychology, Public Health

\section{Keywords}

Mental Health, Mental Disorders, Surveillance, Early Warning

\section{Introduction}

Mental health is a major public health issue that affects economic and social development. At present, mental health problem is a quite severe social problem [1], accounting for about one fifth of total burden of diseases. Some researchers consider that accident or trouble caused by patients with mental disorders ranks the second in causing economic losses, second only to criminal offense [2]. According to estimation of $\mathrm{WHO}$, in China the burden of mental diseases will rise to a quarter of the total burden of diseases in 2020 [3]. Mental disorders are a group of diseases with high recurrence rates, and thus long term maintenance therapy is of great importance to such diseases as treatment interruption or withdrawal will lead to a significant rise in recurrence rates. Therefore, it is necessary to establish a complete mental health service system for treatment and 
recovery of patients with mental disorders. From both longitudinal and horizontal perspectives, the methods for mental health surveillance used in various countries and regions are updating continuously with the adoption of new technologies and shifting from telephone interview to web network and mobile APP software. The method for collecting surveillance data has changed from single machine networking with multiple sentinel points to cloud platform. Moreover, the scope and content of early warning have developed from single factor to whole course early warning. This article provides a review for reference by mental health service personnel.

Severe psychiatric patients, who are the key point of mental health surveillance, have major symptoms like hostility, agitation, suspicion and thought disorder, etc. Sands, et al. [4] found that the risk factors associated with the violence impulsion of such patients are related to these symptoms. The possible aggressive compulsion behaviors of severe psychiatric patients might also be associated with patients' age, gender and positive psychiatric symptoms [5]. Such aggressive behaviors usually occur during the acute phase of psychiatric disease and mainly include various behaviors that threaten and/or harm one's self or other people or objects, manifesting as verbal attack, physical attack and attack on property [6]. Practice has proven that, after symptoms in the acute phase are controlled, most psychiatric patients can return to the society and even participate in appropriate production activities through community-based treatment and rehabilitation [7] [8] [9] [10] [11] and will not do much harm to the society. Moreover, researches have revealed that about $80 \%$ patients with first episode psychosis are likely to recur within 5 years [12] [13], causing severe impairment of social function to the patients, in particular, unemployment and, for some patients, increase in risk of suicide [14] [15] [16]. Therefore, it is necessary to establish a scientific and efficient surveillance and early warning system to implement early surveillance, assessment and warning of psychiatric diseases and keep them under control in the early stage.

\section{Mental Health Surveillance and Early Warning}

Mental health surveillance system covers four major aspects, namely, mental disorder surveillance and early warning, psychological and behavioral problems, risk factors, and mental health service and resources [17]. China did not formally initiate mental health surveillance until the Law on Mental Health was promulgated on May 1, 2013. The key population for surveillance is patients with severe psychosis. The surveillance system includes high-risk single factors into objects of early warning, for example, early warning of compulsion, trouble-making behaviors and suicide. The system can even monitor the whole disease course of psychiatric patients and changes in their mental symptoms and collect important node data in patients' disease period such as medicine administration, dispensing records, follow-up visit records, clinic records and departure records to establish a logical data model, which can be used for analyzing and monitoring 
the mental status of patients in their whole disease course. The model can provide early warning and reminding of any identified risk factors to further guide the diagnosis and treatment of mental disorders. For patients with high risks, network management can be established to strengthen supervision of follow-up visits.

\subsection{Methods of Surveillance and Early Warning}

\subsubsection{Telephone Follow-Up Surveillance}

In the USA, National Health Interview Survey (NHIS) began to be implemented in very early time [18]. NHIS is carried out by means of manual telephone survey and is the most traditional and convenient way to collect information related to schizophrenic patients including their mental conditions, medicine administration and social function. In this survey, professionals sort and record information obtained from telephone interviews. These information can be used to assess patients' mental conditions and timely identify fluctuation of patients' mental conditions. In some region of China, due to condition limitations, telephone survey is used as the primary method for surveillance. However, records obtained from telephone survey are relatively simple and contain limited information of patients, and some information collected via telephone interview is not true or contains unpleasant words, leading to lower reliability of data.

\subsubsection{Outpatient Interview Surveillance}

Outpatient interview surveillance is one of the main methods used for monitoring mental diseases and the primary way to confirm and assess mental disorders. With this method, diagnosis, assessment and early warning of psychiatric patients are based on clinic records maintained by outpatient psychiatrists, who can relatively accurately assess and record the conditions of patients on the basis of interviews, psychiatric examinations and assessment scale. Those patients with high risk are identified with special marks and given treatment advice or rehabilitation guidance. Thus information recorded by psychiatrists are highly reliable and professional. Nonetheless, assessment with this method has certain limitations. Records maintained by psychiatrists are affected by the number of patients and peak of patients visit. Outpatient follow-up interview in peak hours will occupy a lot of time of psychiatrists and the efficiency and quality of assessment will be affected.

\subsubsection{Web-Based Surveillance}

Along with the extensive use of the internet, patients follow-up assessments are placed on the internet and patients can login and submit their assessments at any time, without being affected by time or space limitations. The results of assessments are fed back to the terminals of mental health prevention authority and psychiatrists and mental symptoms of patients are predicted or assessed on the basis of patients self-assessment. Researches [19] have shown that on-line disease screening has the same reliability for specific psychiatric diseases as traditional questionnaire survey does. Such on-line approach of assessment can 
dramatically save labor costs and resources.

\subsubsection{Mobile Phone APP-Based Surveillance}

Increasing evidences show that mobile phones can play an important role in medical care service, particularly in mental health service. In combination with traditional approaches via the internet, mobile phone has achieved significant results in remote consultation [20] and monitoring support [21]. Through mobile APP, patients can receive reminds to go to see doctors, assess their own mental conditions by using appropriate scales at any times and acquire on-line knowledge about mental health related to their own diseases. Compared with Web pages, mobile phone is easier to carry and operate, has a stronger applicability and can be integrated into the daily life of patients [22]. In the future, mobile phone APP may work with wearable devices to collect valuable physical information so as to improve the accuracy of diagnosis and early warning.

\section{The Value of Mental Health Surveillance and Early Warning}

\subsection{Scientific Research Value}

Mental health surveillance involves with a wide range of data. As the scope of mental health surveillance covers both healthy population and unhealthy population, the surveillance data have a very high value for scientific researches. They can be used to study the epidemiological status of specific mental disorders, explore patients' trouble-making warning model and guide emergency response to public health incidents of mental disorders. For these researches, the mental health surveillance system provides excellent research samples, research objects and important data.

\subsection{Economic Value}

The mental health surveillance system can monitor the disease risk propensity of patients. With a complete psychosis prevention information system, various problems of psychiatric patients occurring in the course of rehabilitation can be analyzed and the patients can be informed and advised to timely go to see doctors so as to reduce disease fluctuation and recurrence, control disease condition under a low level and cut medical care costs for patients. To administrative authorities and medical institutions, early identification and early intervention may help reduce consumption of medical resources, decrease the rate of patients hospitalization, lower hospitalization burdens and reasonably allocate medical resources.

\subsection{Decision-Making and Social Effect}

Mental health surveillance data are of great significance and value to early screening, diagnosis, intervention and treatment of psychiatric diseases, embodying people-centered total psychological health service. In particular, it is a public focus in regard to growth of teenagers and support of the old. Moreover, 
mental health surveillance data are also used in clinic assessment to early screen out, through information and artificial intelligence processing, those mental disorder patients with unstable conditions, impulsion, treatment failure, propensity to make trouble and cause accident as an early warning. Such early warning information not only reminds psychiatrists and but also be pushed to superior administration authorities in real time to enable them to follow up the conditions of patients in the whole course of disease and complete emergency response measures. Such surveillance and early warning data also provide a scientific basis for future decisions on mental health.

\section{Perspective}

In summary, as the Law of the People's Republic of China on Mental Health formally came into force, mental health surveillance work has been put into agenda in China. Surveillance and early warning of mental health, as a guarantee of mental health of the whole people and an issue of public health, are paid more and more attention. According to report abroad, many countries have incorporated mental health work into public health systems and are paying more attention to it. A review of the development of mental health surveillance work home and abroad show that this work covers mental disorder, psychological health, disease factors and health care service. The methods used for surveillance have gradually shifted toward modern information technologies: from telephone follow-up surveillance and outpatient interview surveillance to web-based on-line surveillance and mobile phone APP-based surveillance. Analysis of surveillance data also has changed from single point monitoring multiple sentinel points to cloud data sharing. More and more detailed mental health surveillance serves prevention and treatment of mental disorder in the early stage to ensure early identification of the signs of mental disorder and make early intervention possible. Moreover, with increasingly intelligent early warning methods, more effective information can be obtained and pushed to related authority and personnel at real time to allow timely response to emergency and reduce the occurrence of trouble and accidents caused by psychiatric patients. This is of great significance to the maintenance of social order, stability and harmony. Based on this review and mature foreign experiences, we believe that more convenient surveillance methods will be developed in the near future, and combined with artificial intelligence and data models with high operability, an internationally leading mental health surveillance system will be gradually developed in China.

\section{Funding}

This work was supported by a grant from Ningbo Leading Talents Cultivation Program (grant No. NBLJ201802012).

\section{Conflicts of Interest}

The authors declare no conflicts of interest regarding the publication of this paper. 


\section{References}

[1] Li, S.Y. (2010) Attach Importance to the Health Administration of Severe Mental Patients. Journal of Public Health and Preventive Medicine, 21, 1-4.

[2] Zhang, M.Y. (2002) Prevention and Rehabilitation of Mental Illness. Huaxia Press, Beijing.

[3] Ministry of Health P.R. China (2007) China Health Statistics Yearbook 2007. China Union Medical University Press, Beijing.

[4] Sands, N., Elsom, S., Gerdtz, M. and Khaw, D. (2012) Mental Health-Related Risk Factors for Violence: Using the Evidence to Guide Mental Health Triage Decision Making. Journal of Psychiatric and Mental Health Nursing, 19, 690-701. https://doi.org/10.1111/j.1365-2850.2011.01839.x

[5] Guo, X.Z., Xi, D.B., Li, X.L., et al. (2006) Related Factors and Nursing Effect of Aggressive Behavior in Newly Admitted Patients with Schizophrenia. Chinese Journal of Behavioral Medical Science, 15, 752-753.

[6] Morrbam, E.F. (1990) Violent Psychiatric Inpatients in a Public Hospital. Scholarly Inquiry for Nursing Practice, 4, 65-86.

[7] Killaspy, H. (2006) From the Asylum to Community Care: Learning from Experience. British Medical Bulletin, 79-80, 245-258. https://doi.org/10.1093/bmb/ldl017

[8] Bernabei, R., Landi, F., Gambassi, G., et al. (1998) Randomised Trial of Impact of Model of Integrated Care and Case Management for Older People Living in the Community. British Medical Journal, 316, 1348-1351.

https://doi.org/10.1136/bmj.316.7141.1348

[9] Dieterich, M., Irving, C.B., Park, B. and Marshall, M. (2010) Intensive Case Management for Severe Mental Illness. Cochrane Database of Systematic Reviews, 10-17. https://doi.org/10.1002/14651858.CD007906.pub2

[10] Franklin, J.L., Solovitz, B., Mason, M., Clemons, J.R. and Miller, G.E. (1987) An Evaluation of Case Management. American Journal of Public Health, 77, 674-678.

https://doi.org/10.2105/AJPH.77.6.674

[11] Godldy, S.H., Finch, M., Dougan, L., et al. (2000) Case Management for Dually Diagnosed Individuals Involved in the Criminal Justice System. Journal of Substance Abuse Treatment, 18, 137-148. https://doi.org/10.1016/S0740-5472(99)00027-6

[12] Robinson, D., Woerner, M.G., Alvir, J.M., Bilder, R., Goldman, R., Geisler, S., et al. (1999) Predictors of Relapse Following Response from a First Episode of Schizophrenia or Schizoaffective Disorder. Archives of General Psychiatry, 56, 241-247. https://doi.org/10.1001/archpsyc.56.3.241

[13] Alvarez-Jimenez, M., Priede, A., Hetrick, S.E., Bendall, S., Killackey, E., Parker, A.G., et al. (2012) Risk Factors for Relapse Following Treatment for First Episode Psychosis: A Systematic Review and Meta-Analysis of Longitudinal Studies. Schizophrenia Research, 139, 116-128. https://doi.org/10.1016/j.schres.2012.05.007

[14] Appleby, L. (1992) Suicide in Psychiatric Patients: Risk and Prevention. The British Journal of Psychiatry, 161, 749-758. https://doi.org/10.1192/bjp.161.6.749

[15] Iqbal, Z., Birchwood, M., Chadwick, P. and Trower, P. (2000) Cognitive Approach to Depression and Suicidal Thinking in Psychosis. 2. Testing the Validity of a Social Ranking Model. The British Journal of Psychiatry, 177, 522-528.

https://doi.org/10.1192/bjp.177.6.522

[16] Birchwood, M., Iqbal, Z., Chadwick, P. and Trower, P. (2000) Cognitive Approach to Depression and Suicidal Thinking in Psychosis. 1. Ontogeny of Post-Psychotic Depression. The British Journal of Psychiatry, 177, 516-521. 
https://doi.org/10.1192/bjp.177.6.516

[17] Zhou, W. and Xiao, S.Y. (2014) Development of Mental Health Surveillance in Foreign Countries. Chinese Mental Health Journal, 28, 3.

[18] Reeves, W.C., Strine, T.W., Pratt, L.A., et al. (2011) Mental Illness Surveillance among Adults in the United States. MMWR Surveillance Summaries, 60, 1-29.

[19] Hirsch, O., Hauschild, F., Schmidt, M.H., Baum, E. and Christiansen, H. (2013) Comparison of Web-Based and Paper-Based Administration of ADHD Questionnaires for Adults. Journal of Medical Internet Research, 15, 47.

https://doi.org/10.2196/jmir.2225

[20] Hilty, D.M., Ferrer, D.C., Parish, M.B., Johnston, B., Callahan, E.J. and Yellowlees, P.M. (2013) The Effectiveness of Telemental Health: A 2013 Review. Telemedicine and e-Health, 19, 444-454. https://doi.org/10.1089/tmj.2013.0075

[21] Mundt, J.C., Greist, J.H., Gelenberg, A.J., Katzelnick, D.J., Jefferson, J.W. and Modell, J.G. (2010) Feasibility and Validation of a Computer-Automated ColumbiaSuicide Severity Rating Scale Using Interactive Voice Response Technology. Journal of Psychiatric Research, 44, 1224-1228. https://doi.org/10.1016/j.jpsychires.2010.04.025

[22] Gonzales, R., Ang, A., Murphy, D., Glik, D. and Anglin, M. (2014) Substance Use Recovery Outcomes among a Cohort of Youth Participating in a Mobile-Based Texting Aftercare Pilot Program. Journal of Substance Abuse Treatment, 47, 20-26. https://doi.org/10.1016/j.jsat.2014.01.010 